\title{
Cloud droplets in a bulk formulation and its application to buoyancy reversal instability
}

\author{
A. de Lozar* and J. P. Mellado \\ Max Planck Institute for Meteorology, Hamburg, Germany \\ ${ }^{*}$ Correspondence to: A. de Lozar, Max Planck Institute for Meteorology, 20146 Hamburg, Germany. E-mail: \\ adelozar@googlemail.com
}

\begin{abstract}
Mixing processes at the boundary of clouds often include typical length-scales of several metres. Such length-scales are too large for current Lagrangian models but they are also poorly resolved by typical Eulerian-based large-eddy simulations. Here, a bulk formulation is introduced for direct numerical simulations. Two main assumptions sustain this approach: the continuum approximation and the liquid-phase diffusion approximation. The formulation includes the small-scale features that originate from microscopic droplet dynamics: sedimentation, finite-time condensation/evaporation, inertial effects and the low diffusion of liquid droplets with respect to vapour. The methodology is applied to the study of the buoyancy reversal instability that occurs at the top of stratocumulus clouds as a consequence of evaporative cooling. The inclusion of sedimentation, low liquid-phase diffusion and finite-time evaporation have a negative impact on instability when compared with the equilibrium formulation. The combined effect of all these small-scale features reduces mixing at the cloud top by at least $90 \%$. This strong reduction is explained by a condensate-free middle layer, which emerges when droplets leave the cloud interface due to sedimentation.
\end{abstract}

Key Words: turbulence; cloud microphysics; direct numerical simulations; cloud boundary; stratocumulus; cloud-top entrainment instability; buoyancy reversal instability

Received 18 April 2013; Revised 19 July 2013; Accepted 26 July 2013; Published online in Wiley Online Library 5 September 2013

\section{Introduction}

The rate at which a cloud entrains dry air determines the cloud lifetime and is thus a key parameter for modelling clouds. However, estimations of entrainment are difficult because the mixing is turbulent and involves a wide range of length-scales, from the cloud size down to the Kolmogorov length. Additionally, short time-scale processes related to the microscopic droplet dynamics are usually ignored in large-scale models but might also play an important role for the mixing. For example, thermodynamic equilibrium is usually assumed, but in reality droplets require a finite time to reach this equilibrium, allowing for small supersaturated and subsaturated regions (Vaillancourt et al., 2001). Also, droplets are not equally distributed and tend to concentrate preferentially in regions of low vorticity (Shaw, 2003). Although the liquid water content in a cloud is small ( $\sim 1 \%$ of the total mass), the combined effect of these small-scale processes is believed to be important not only for mixing but also for rain formation, radiation and the interaction of the cloud with aerosols (Bodenschatz et al., 2010; Grabowski and Wang, 2013). However, quantitative predictions are not yet accurate enough. This is partly due to the wide separation between the scales at which these microphysical processes take place and the largest mixing scales (Devenish et al., 2012). Most of the current models aim at either a faithful representation of small-scale processes at the centimetre scale (Andrejczuk et al., 2009; Kumar et al., 2012) or a good representation of the cloud scales at the kilometre scale (Ackerman et al., 2009). In this article, we propose a formulation for warm clouds that combines the exactitude of direct numerical simulations (DNS) of turbulent flows with the simplicity of bulk formulations of cloud droplets. The goal is to provide a good description of small-scale processes while still allowing for domains of several metres, supposedly the scale at which cloud mixing occurs (Bodenschatz et al., 2010).

In bulk models (also called two-fluid formulations in some literature), the collection of droplets inside a control volume is characterized by the mass density of liquid and the velocity of the centre of mass of the droplets. When the number of droplets in the control volume is high enough, the density and velocity are well described by continuous functions, allowing for an Eulerian description of the flow (Marble, 1970). Due to their simplicity, bulk models are very efficient and allow for simulations of large domains. One important limitation is that details of the droplet size spectrum are not considered in the model. This is a big source of uncertainty for radiation schemes because they rely on knowledge of the droplet radius across the cloud (Chosson et al., 2007). Further limitations are as follows: collision schemes are coarse (but efficient) (Seifert and Beheng, 2006); the assumption of equilibrium thermodynamics may be inappropriate (Grabowski and Smolarkiewicz, 1990); and preferential 
concentration effects and sedimentation are often ignored (Shaw, 2003).

In a Lagrangian formulation, the equations of motion are solved for each cloud droplet (Vaillancourt et al., 2001, 2002; Lanotte et al., 2009; Kumar et al., 2013). The main advantage of this methodology is that it allows for a faithful representation of small-scale cloud features. The main drawback is that the domain size is severely limited by the number of droplets that computer clusters can handle. Current simulations include $\sim 10^{8}$ droplets, which corresponds to a $\sim 30 \mathrm{~cm}^{3}$ domain for typical cloud-number densities $\left(\sim 100 \mathrm{~cm}^{-3}\right)$. Communication between computer cores seems to be the bottleneck because droplets move across the whole domain, making efficient parallelization difficult.

An alternative to the formulations introduced above is to use a statistical approach in which the description of the droplets is given by a probability distribution function (pdf: Williams, 1985). As with the bulk formulation, the statistical description is applicable when the number of droplets in each control volume is high. Such a formulation allows for an Eulerian description of the liquid phase while retaining droplet size spectra. However, an accurate statistical formulation is computationally very expensive because the distribution function has to be calculated at each grid box. Two main simplifying strategies are used. In the first approach, the distribution function is assumed to have a particular functional form, derived from previous observations or experiments (Seifert and Beheng, 2006). In general, the functional form depends on only a few parameters or moments for which evolution equations are provided (Ziemer and Wacker, 2012). In the second approach, the distribution function is discretized in a set of discrete bins (Andrejczuk et al., 2004). This approach is generally more expensive but has the advantage that it is more flexible and does not depend on any hypothesis for the functional form.

The advantage of a statistical formulation is that it provides some freedom to choose the ratio of efficiency to accuracy. On the one hand, a simulation using the Lagrangian formulation is equivalent to a simulation using a pdf formulation where the pdf is perfectly parametrized (using an infinite number of bins for example) if the number of droplets is high enough for a statistical approach. On the other hand, the bulk formulation is recovered when the equation for the distribution function is integrated to an equation for a single scalar, typically the total mass of liquid. Therefore, varying the parametrization of the pdf allows us to move between different formulations.

Apart from the different strategies to describe the droplets, models also differ in how they solve the turbulent field. The most exact method is DNS. In DNS, the Navier-Stokes equations are solved without any parametrization so that even the smallest eddies are resolved (Moin and Mahesh, 1998). Therefore, no assumptions about the turbulent field are necessary. The main drawback is the limitation that DNS imposes on domain size. In clouds, the Kolmogorov scale is of the order of 1-5 mm (Vaillancourt and Yau, 2000), which implies that simulation domains can only reach an order of $10 \mathrm{~m}$ with current computational capabilities. This limitation can be overcome using turbulent models like large-eddy simulations (LES). In LES models, eddies are resolved up to the grid-box scale (circa $10 \mathrm{~m}$ ) while turbulence at the subgrid scale is parametrized using different subgrid models (Smagorinsky, 1963; Deardorff, 1973). However, it is uncertain how the parametrization of subgrid turbulence and other subgrid processes might influence the final results.

In general, there is a tendency to use LES with bulk models and DNS with Lagrangian models. Following this trend, there are also some studies that use the pdf formulation together with an intermediate DNS/LES model (Andrejczuk et al., 2004). This is justified because coarse models attempt to solve large-scale problems, whereas the more exact formulations aim at the smaller scales. Nonetheless, other combinations are also possible. At one end of the scale, Lagrangian models can be implemented in LES to model the droplet dynamics (Andrejczuk et al., 2008). At the other end, Mellado et al. (2010b) combined a pure bulk formulation with a DNS approach in order to simulate domains larger than in any Lagrangian calculation, while still resolving all scales of the flow. Using this formulation, Mellado (2010) investigated the buoyancy reversal instability (BRI) and found that the turbulence generated by evaporative cooling alone is too weak to break the stratocumulus deck and therefore cannot alone explain cloud-top entrainment instability (Randall, 1980; Deardorff, 1980; Wood, 2012). That study was aimed at providing an upper limit for BRI and did not consider any small-scale features from the droplet dynamics, under the assumption that one would just reduce the mixing and therefore the turbulence intensity at the cloud-top (Mellado et al., 2010a).

Based on that previous work, we develop a formulation for warm, non-precipitating clouds where finite-time thermodynamics (supersaturation), particle inertia and settling velocity are included. Chemical reactions, radiation and collisions are outside the scope of this article. In section 2 we write the pdf and transport evolution equations, including the physical processes just mentioned. Next we simplify the non-dimensional equations by assuming a given shape of the pdf with a single parameter, as discussed in section 3 . The resulting approach is equivalent to a bulk formulation. This procedure allows us to examine our simplifying hypothesis and establish the limits of the methodology. As an example, in section 4 we apply the formulation to test the hypothesis that small-scale processes have a negative impact on the BRI in stratocumuli. To this end, we investigate a cloud-top configuration driven solely by evaporative cooling. The properties of the investigated configuration are based on the stratocumulus measurements obtained in the DYCOMS-II campaign (Stevens et al., 2003). Readers that are only interested in the impact of small-scale processes on the BRI can read section 4 directly and avoid the sections that describe the bulk formulation.

\section{General formulation}

Let us consider a small reference volume $\mathrm{d} V$ at a position $\mathbf{x}$ in the cloud. The total mass of the volume, $\mathrm{d} M$, is divided into the different species that form a warm cloud: dry air, $\mathrm{d} M_{\mathrm{D}}$, vapour, $\mathrm{d} M_{\mathrm{V}}$, and liquid water, $\mathrm{d} M_{\mathrm{l}}$. This allows us to define the mass fraction of each component as

$$
q_{\mathrm{l}}=\frac{\mathrm{d} M_{\mathrm{l}}}{\mathrm{d} M}, \quad q_{\mathrm{v}}=\frac{\mathrm{d} M_{\mathrm{V}}}{\mathrm{d} M}, \quad q_{\mathrm{d}}=\frac{\mathrm{d} M_{\mathrm{D}}}{\mathrm{d} M},
$$

such that $q_{\mathrm{l}}+q_{\mathrm{v}}+q_{\mathrm{d}}=1$. It is also useful to define the mass fraction of the gas phase, $q_{\mathrm{g}}=q_{\mathrm{v}}+q_{\mathrm{d}}$, and the mass fraction of total water, $q_{\mathrm{t}}=q_{\mathrm{v}}+q_{1}$. The density of each component is calculated from the total density, $\rho=\mathrm{d} M / \mathrm{d} V$, as $\rho q_{i}$.

In this section we first discuss the two main hypotheses that sustain our framework: the continuum approximation for description of the droplets and diffusion of the liquid phase. Next, the transport equations are summarized and the limitations of the existing equilibrium model are presented, motivating the non-equilibrium formulation described in this article.

\subsection{Continuum formulation}

We consider only cloud droplets of spherical shape (diameter $D<20 \mu \mathrm{m}$ ). Each cloud droplet is thus solely characterized by its radius $r$ and velocity $\mathbf{v}$. This allows us to define the following probability distribution functions:

- $P(\mathbf{x}, r, \mathbf{v}) \mathrm{d} \mathbf{v} \mathrm{d} r \mathrm{~d} \mathbf{x}$ : the probable number of droplets with radius between $r$ and $r+\mathrm{d} r$ and velocity between $\mathbf{v}$ and $\mathbf{v}+\mathrm{d} \mathbf{v}$ in volume $\mathrm{d} V \equiv\|\mathrm{d} \mathbf{x}\|$ at a position $\mathbf{x}$;

- $f(\mathbf{x}, r) \mathrm{d} r \mathrm{~d} \mathbf{x}=\int P(\mathbf{x}, r, \mathbf{v}) \mathrm{d} \mathbf{v}$ : the probable number of droplets with radius between $\mathrm{r}$ and $r+\mathrm{d} r$ and any velocity in volume $\mathrm{d} V$ at a position $\mathbf{x}$; and 
- $n(\mathbf{x}) \mathrm{d} \mathbf{x}=\int f(r, \mathbf{v}) \mathrm{d} r$ : the probable number of droplets in volume $\mathrm{d} V$ at a position $\mathbf{x}$.

We use a continuum formulation for the disperse liquid phase and therefore we assume that the pdfs vary smoothly in space. A continuum formulation is valid when the number of droplets in each reference volume, $\mathrm{d} V$, is large enough. Therefore the validity of the continuum assumption depends on the size of the chosen reference volume. The reference volume is defined by the length-scale of the smallest variations that are resolved in the model. For example, typical volumes in LES of the order of $10^{3} \mathrm{~m}^{3}$ contain around $10^{9}$ droplets (assuming droplet densities in clouds of the order of $100 \mathrm{~cm}^{-3}$ ), which is clearly enough for the continuum hypothesis. In DNS, we aim to resolve variations of the flow of the order of the Kolmogorov scale. In clouds, the Kolmogorov scale is of the order of $\eta \sim 1-5 \mathrm{~mm}$ (Vaillancourt and $\mathrm{Yau}, 2000$ ) and the corresponding droplet concentration in each reference volume is of the order of $\sim 0.1-10$ droplets per reference volume. This low number density casts some doubts over the validity of the formulation, because the continuum hypothesis effectively averages the action of every droplet over several reference volumes. In this sense the continuum scheme is similar, although probably less accurate, than Lagrangian formulations where the action of each droplet is averaged over the neighbouring grid points of the Eulerian field (Kumar et al., 2013). We expect that the continuum formulation provides a good approximation only when there is not a strong nonlinear response of small scales of flow to the fluctuations in droplet concentration. A definitive answer regarding the validity of the formulation will only be given in the event of a direct comparison with experiments or with different Lagrangian schemes.

In a continuum formulation, the conservation law of the number of droplets reads (Vincenti and Kruger, 1967)

$$
\begin{aligned}
\frac{\partial P}{\partial t}= & -\nabla_{\mathbf{x}}(\mathbf{v} P)-\nabla_{\mathbf{v}}\left(P \frac{\mathrm{d} \mathbf{v}}{\mathrm{d} t}\right) \\
& -\frac{\partial}{\partial r}\left(P \frac{\mathrm{d} r}{\mathrm{~d} t}\right)+\hat{\eta}(\mathbf{x}, \mathbf{v}, r)+K
\end{aligned}
$$

where $\hat{\eta}$ and $K$ account for the nucleation and collision rates, respectively, and $\mathrm{d} r / \mathrm{d} t$ accounts for the condensation/evaporation rate of each droplet. For simplicity, we assume dilute conditions and do not consider any collision in this article $(K=0)$.

In this article, we consider properties that depend only on the radius of the droplets and are therefore independent of their velocity. Assuming that $P$ decays rapidly for high velocities, Eq. (2) can be integrated for all velocities (Shaw, 2003):

$$
\frac{\partial f}{\partial t}=-\nabla_{\mathbf{x}}\left(f \mathbf{v}_{\mathbf{r}}\right)-\frac{\partial}{\partial r}\left(f \frac{\mathrm{d} r}{\mathrm{~d} t}\right)+\eta(\mathbf{x}, r),
$$

where $\mathbf{v}_{\mathbf{r}}$ is the mean velocity of droplets with radius $r$ and $\eta(\mathbf{x}, r)$ is the number of particles of radius $r$ nucleated or destroyed per unit of time. The probability distribution function, $f$, relates the microphysical properties of the droplets to the mean bulk properties of the flow. For example, we can integrate the mass of all the droplets to obtain the density of liquid of the flow:

$$
\rho q_{1}=\int_{0}^{\infty} m_{\mathrm{D}} f \mathrm{~d} r=\frac{4}{3} \rho_{\mathrm{l}} n \pi \overline{r^{3}},
$$

where we have used that all droplets are spherical with mass $m_{\mathrm{D}}=(4 / 3) \pi \rho_{\mathrm{l}} r^{3}$. Note that we have reserved $\rho_{\mathrm{l}}$ for the density of the liquid phase and that the density of liquid water in the cloud is defined as $\rho q_{1}$. The overbar denotes the mean value of a property, defined as

$$
\bar{A}(\mathbf{x})=\frac{1}{n} \int_{0}^{\infty} A(\mathbf{x}, r) f(\mathbf{x}, r) \mathrm{d} r .
$$

\subsection{Relative velocities}

One of the main difficulties of the cloud problem is that the different species in a cloud typically move with different velocities. In general, we refer to the velocity of a species as the velocity of the centre of mass of all its molecules or droplets contained in the reference volume. We refer to the velocity of the dry, vapour and liquid phase as $\mathbf{V}_{\mathrm{d}}, \mathbf{V}_{\mathrm{v}}$ and $\mathbf{V}_{\mathrm{l}}$, respectively. The mean velocity is thus

$$
\mathbf{u}=q_{\mathrm{d}} \mathbf{V}_{\mathrm{d}}+q_{\mathrm{v}} \mathbf{V}_{\mathrm{v}}+q_{\mathrm{l}} \mathbf{V}_{\mathrm{l}} .
$$

Consistently, we define the velocity of the gas phase as $q_{\mathrm{g}} \mathbf{V}_{\mathrm{g}}=q_{\mathrm{d}} \mathbf{V}_{\mathrm{d}}+q_{\mathrm{v}} \mathbf{V}_{\mathrm{v}}$. The liquid-phase velocity can be expressed as a function of the mean velocities of the individual droplets using the probability distribution functions described above:

$$
q_{\mathrm{l}} \mathbf{V}_{\mathrm{l}}=\frac{4 \pi \rho_{\mathrm{l}}}{3 \rho} \int r^{3} f(r) \mathbf{v}_{\mathrm{r}}(r) \mathrm{d} r .
$$

We assume that the drift velocities of the vapour and dry air are of diffusive nature, caused by the Brownian motion of the molecules. We model diffusion using Fick's law (Williams, 1985), which allows us to write the drift velocities of the vapour and the dry air as

$$
q_{i} / q_{\mathrm{g}}\left(\mathbf{V}_{i}-\mathbf{V}_{\mathrm{g}}\right)=-\kappa_{\mathrm{v}} \nabla\left(q_{i} / q_{\mathrm{g}}\right),
$$

where the index $i$ refers to the vapour and dry air phases and $\kappa_{\mathrm{v}}$ is the diffusion coefficient of the binary mixture formed by vapour and dry air. The hypothesis underlying Eq. (8) is that the diffusion of dry air and vapour behaves as if the droplets were not present. This hypothesis is justified by the small volume occupied by the droplets. From Eq. (8), we calculate the relative velocity of the vapour phase over the dry air phase:

$$
\mathbf{V}_{\mathrm{v}}-\mathbf{V}_{\mathrm{d}}=-\kappa_{\mathrm{v}} \nabla \ln \left(q_{\mathrm{v}} / q_{\mathrm{d}}\right) .
$$

The calculation of the drift velocity of the liquid phase is more complex due to inertia and gravitational effects. We divide the total drift velocity into two components: one due to diffusion and a second one that encompasses all other possible sources for the droplet drift, like inertia or sedimentation. This allows us to write the liquid-phase drift velocity with respect to the gas phase as

$$
\mathbf{V}_{\mathrm{l}}-\mathbf{V}_{\mathrm{g}}=-\kappa_{\mathrm{l}} \nabla \ln \left(q_{\mathrm{l}} / q_{\mathrm{g}}\right)+\mathbf{D}
$$

where $\mathbf{D}$ is the non-diffusional drift velocity:

$$
\rho q_{\mathrm{l}} \mathbf{D}=\frac{4}{3} \pi \rho_{\mathrm{l}} \int r^{3} f(r)\left(\mathbf{v}_{\mathrm{r}}^{\mathrm{D}}(r)-\mathbf{V}_{\mathrm{g}}\right) \mathrm{d} r,
$$

and $\mathbf{v}_{\mathrm{r}}^{\mathrm{D}}$ is the mean velocity of droplets of radius $r$ once diffusion is removed.

In general, thermal fluctuations for particles of the size of droplets (which imply $\kappa_{1}=10^{-12} \mathrm{~m}^{2} \mathrm{~s}^{-1}$ in Mellado et al., 2010b) are much smaller than thermal fluctuations in the gaseous phase (which imply $\kappa_{\mathrm{v}}=10^{-5} \mathrm{~m}^{2} \mathrm{~s}^{-1}$ ). Therefore, the thermal diffusion of the liquid phase can be safely neglected when compared with the molecular diffusion of the vapour. We retain the liquidphase diffusion term in the formulation because simulations with a realistic value of $\kappa_{l}$ require an extremely high numerical resolution. A higher, unphysical, liquid-phase diffusion allows us to study a much wider range of problems, which would otherwise be too expensive computationally. In order to check the effect of the unphysical diffusion on each problem, it is then necessary to compare cases with different values of $\kappa_{1}$.

Combining Eqs (6), (9) and (10), we obtain the following expressions for the velocities of each species:

$$
\begin{aligned}
& \mathbf{V}_{\mathrm{d}}=\mathbf{u}-\kappa_{\mathrm{v}} \nabla \ln \left(q_{\mathrm{d}} / q_{\mathrm{g}}\right)-\kappa_{\mathrm{l}} \nabla \ln \left(q_{\mathrm{g}}\right)-q_{\mathrm{l}} \mathbf{D}, \\
& \mathbf{V}_{\mathrm{v}}=\mathbf{u}-\kappa_{\mathrm{v}} \nabla \ln \left(q_{\mathrm{v}} / q_{\mathrm{g}}\right)-\kappa_{\mathrm{l}} \nabla \ln \left(q_{\mathrm{g}}\right)-q_{\mathrm{l}} \mathbf{D}, \\
& \mathbf{V}_{\mathrm{l}}=\mathbf{u}-\kappa_{\mathrm{l}} \nabla \ln \left(q_{\mathrm{l}}\right)+q_{\mathrm{g}} \mathbf{D} .
\end{aligned}
$$




\subsection{Transport equations}

So far, we have introduced a continuum description of a disperse and dilute two-phase flow. The next set of equations reflects the conservation of mass of the different constituents, as well as momentum and energy for the total mixture:

$$
\begin{aligned}
& \partial \rho / \partial t(\rho)+\nabla \cdot(\rho \mathbf{u})=0 \\
& \partial / \partial t\left(\rho q_{\mathrm{t}}\right)+\nabla \cdot\left(\rho q_{\mathrm{t}} \mathbf{u}\right)=-\nabla \cdot \mathbf{j}_{\mathrm{t}} \\
& \partial / \partial t\left(\rho q_{1}\right)+\nabla \cdot\left(\rho q_{1} \mathbf{u}\right)=-\nabla \cdot \mathbf{j}_{1}+C+N \\
& \partial / \partial t(\rho \mathbf{u})+\nabla \cdot(\rho \mathbf{u u})=-\nabla p+\nabla \cdot \tau+R i \frac{\rho}{\Delta \rho / \rho_{0}} \mathbf{e}_{\mathrm{g}}, \\
& \partial / \partial t(\rho e)+\nabla \cdot(\rho e \mathbf{u})=-\left(\gamma_{0}-1\right) M^{2} p \nabla \cdot \mathbf{u} \\
& \quad+\left(\gamma_{0}-1\right) M^{2} \tau: \nabla \mathbf{u}-\nabla \cdot \mathbf{j}_{\mathrm{q}},
\end{aligned}
$$

where $C$ and $N$ represent the condensation/evaporation and nucleation terms, respectively. In Appendix A, we summarize how to obtain the drift transport terms on the right-hand sides of Eq. (13) from the drift velocities presented in Eq. (12). The result of these calculations read

$$
\begin{aligned}
-\mathbf{j}_{\mathrm{t}}= & \frac{\mu}{\operatorname{Re} S c}\left[\nabla q_{\mathrm{t}}\right. \\
& \left.+\left(\frac{S c}{S c_{l}}-1\right) \frac{q_{\mathrm{d}}}{q_{\mathrm{g}}} \nabla q_{\mathrm{l}}\right]-\left(1-q_{\mathrm{t}}\right) \rho q_{\mathrm{l}} \mathbf{d}, \\
-\mathbf{j}_{\mathrm{l}}= & \frac{\mu}{\operatorname{Re} S c_{\mathrm{l}}} \nabla q_{1}-\rho q_{1} q_{\mathrm{g}} \mathbf{d}, \\
\tau / \mu= & \frac{1}{\operatorname{Re}}\left[\nabla \mathbf{u}+(\nabla \mathbf{u})^{\mathrm{T}}-\frac{2}{3}(\nabla \cdot \mathbf{u}) I\right], \\
-\mathbf{j}_{\mathrm{q}}= & \frac{\mu}{\operatorname{Re} P r}\left[\nabla h+\left(\frac{P r}{S c}-1\right)\left(h_{\mathrm{d}} \nabla q_{\mathrm{d}}+h_{\mathrm{v}} \nabla q_{\mathrm{v}}\right.\right. \\
& \left.\left.+h_{\mathrm{l}} \nabla q_{1}\right)+\left(\frac{P r}{S c_{\mathrm{l}}}-\frac{P r}{S c}\right)\left(h_{1}-h_{\mathrm{g}}\right) \nabla q_{\mathrm{l}}\right] \\
& -\left(h_{1}-h\right) \rho q_{1} \mathbf{d} .
\end{aligned}
$$

For generality, the equations have been non-dimensionalized with reference values for velocity, $U_{0}$, length, $L_{0}$, temperature, $T_{0}$, density, $\rho_{0}$, heat capacity, $c_{\mathrm{p}, 0}$, and dynamic viscosity, $\mu_{0}$. Accordingly, the pressure is scaled by $\rho_{0} U_{0}^{2}$, the time by $t_{0}=L_{0} / U_{0}$ and the enthalpy $h$ and internal energy $e$ by $c_{\mathrm{p}, 0} T_{0}$. The non-dimensional drift velocity is defined as $\mathbf{d}=\mathbf{D} / U_{0}$.

The reference non-dimensional numbers that appear in the equations are

$$
\begin{array}{cc}
R e=\frac{U_{0} L_{0}}{\mu_{0} / \rho_{0}}, \quad R i=\frac{\Delta \rho g L_{0}}{\rho_{0} U_{0}^{2}}, \\
M=\frac{U_{0}}{\sqrt{\gamma_{0} R_{\mathrm{d}} T_{0}}}, \quad Q=\frac{\Delta e_{\mathrm{lv}}^{0}}{c_{\mathrm{p}, 0} T_{0}}, \Delta \rho / \rho_{0},
\end{array}
$$

where $\Delta \rho$ is a typical density difference of the problem, $\Delta e_{\mathrm{lv}}^{0}$ is the latent heat of vaporization at $0 \mathrm{~K}, R e$ is the Reynolds number, $R i$ is the Richardson number, $M$ is the Mach number and $Q$ is a non-dimensional latent heat parameter. Three non-dimensional numbers describe the ratio of molecular diffusion of momentum to the diffusion of the different mass species and heat: two Schmidt numbers for the molecular transport of vapour and liquid and a Prandtl number for the transport of heat,

$$
S c=\mu /\left(\rho \kappa_{\mathrm{v}}\right), \quad S c_{1}=\mu /\left(\rho \kappa_{1}\right), \quad \operatorname{Pr}=\mu /\left(\lambda / c_{\mathrm{p}}\right) .
$$

In addition, the non-dimensional equation of state and thermodynamics relations for the internal energy and enthalpy are

$$
\begin{aligned}
\gamma_{0} M^{2} p / \rho & =T\left[1+q_{\mathrm{t}}\left(R_{\mathrm{v}} / R_{\mathrm{d}}-1\right)-q_{\mathrm{l}} R_{\mathrm{v}} / R_{\mathrm{d}}\right], \\
e & =\left[\left(q_{\mathrm{t}}-q_{1}\right) c_{\mathrm{v}, \mathrm{v}}+\left(1-q_{\mathrm{t}}\right) c_{\mathrm{v}, \mathrm{d}}+q_{1} c_{\mathrm{l}}\right] T-q_{\mathrm{l}} Q, \\
h_{i} & =e_{i}+\left(\gamma_{0}-1\right) M^{2} p_{i} / \rho_{i},
\end{aligned}
$$

where the reference ratio of specific heats is defined by $\gamma_{0}-1=1 /\left(c_{\mathrm{p}, 0} / R_{\mathrm{d}}-1\right)$ and $R_{\mathrm{d}} / c_{\mathrm{p}, 0}$ and $R_{\mathrm{v}} / c_{\mathrm{p}, 0}$ are the nondimensional dry air and vapour gas constants. This form of the equation of state is obtained when taking the next assumptions: Dalton's law, both the vapour and dry air phases obey the ideal gas equation and the volume fraction of liquid is negligible. Notice that in the case of the fully compressible formulation the pressure in the equation of state is the same as the dynamical pressure in the Navier-Stokes equations (Eq. (13)).

Details about the derivation of the previous governing equations and the assumptions needed in the process can be found in Mellado et al. (2010b). First, thermal equilibrium has been assumed in order to define one single temperature of the fluid mixture and be able to use the thermodynamical relations in Eq. (17). This simplification is quite exact due to the low liquid concentrations in clouds $\left(q_{1} \sim 10^{-3}\right)$. Even during phase transitions, when the temperature difference between a droplet and the surrounding air can be as large as $5 \mathrm{~K}$ (Rogers and Yau, 1989), the differences of the air temperature from thermal equilibrium remain below $0.02 \mathrm{~K}$. Equally, dilute conditions have been used in order to simplify additional terms in the transport equations. Hence, the major effect of the droplets on the flow dynamics that is retained in this formulation is the latent heat effect and the heterogeneity associated with it due to the relative motion of the liquid phase with respect to the gas phase.

\subsection{Liquid water in equilibrium}

In addition, the equilibrium formulation further developed in Mellado et al. (2010b) invokes mechanical and phase equilibrium. The former corresponds to the limit of vanishing drift velocity $(\mathbf{d}=0)$, greatly simplifying the transport equations. The latter implies that the change of phase from liquid water to vapour and the reverse transition are infinitely fast. The liquid water $q_{1}$ is then uniquely given by the state variables as

$$
q_{1}=q_{1, \mathrm{eq}}\left(\rho, e, q_{\mathrm{t}}\right)
$$

and there is no need to retain a prognostic equation for $q_{1}$, nor to model the nucleation and condensation terms.

In the case in which liquid water is present $\left(q_{1}>0\right)$, Eq. (18) is solved using the condition that water at the vapour phase is at the vapour pressure: $q_{\mathrm{v}}=q_{\mathrm{v}}^{\text {sat }}=p_{\mathrm{S}}(T) /\left(\rho R_{\mathrm{V}} T\right)$, where the saturation pressure, $p_{\mathrm{S}}(T)$, is obtained from an empirical formula. This is a well known approach (Emanuel, 1994) and is not repeated here. As in Mellado et al. (2010b), we smooth discontinuous gradients in Eq. (18) over a distance $\delta_{\mathrm{s}}$ in thermodynamic space. It was also shown that, when this distance is small $\left(\delta_{\mathrm{s}} \lesssim 1 / 100\right)$, the flow statistics of interest are independent of this factor.

\section{Non-equilibrium formulation}

The assumption of equilibrium is very useful to close the system and therefore it is often employed. However, droplets in clouds require several seconds to evaporate or grow $(\sim 10 \mathrm{~s}$ for $r=$ $5 \mu \mathrm{m}$ ), making the phase equilibrium hypothesis questionable in certain situations. For instance, if droplets at the cloud interface move fast enough then big regions out of equilibrium might be the norm, alternating liquid water in undersaturated parcels with supersaturated regions (Shaw, 2003; Andrejczuk et al., 2004; Burnet and Brenguier, 2007). The mechanical equilibrium assumption might be also questionable in certain situations because it neglects sedimentation. In many stratocumulus clouds, the settling velocity $\left(\sim 3 \mathrm{~mm} \mathrm{~s}^{-1}\right.$ for $\left.r=5 \mu \mathrm{m}\right)$ is comparable to the scales representative of the mixing, such as the entrainment velocity, and the settling can significantly change the mixing behaviour (Ackerman et al., 2004; Bretherton et al., 2007).

If thermodynamics and mechanical equilibrium are not a valid approximation, then it is necessary to model the small-scale processes that come from the microscopic droplet dynamics, in 
particular condensation/evaporation, inertial drift, settling and nucleation. The condensation/evaporation and nucleation terms are calculated by taking the time derivative of Eq. (4) and then using Eqs (3) and (12):

$$
\begin{aligned}
& C=3 q_{1}^{0} n r^{2} \frac{\mathrm{d} r}{\mathrm{~d} t}, \\
& N=q_{1}^{0} \int r^{3} \eta(r) \mathrm{d} r
\end{aligned}
$$

where the radius of the droplets has been normalized by a typical radius $r_{0}$, the concentration of particles by a typical concentration $n_{0}$, the nucleation term by $n_{0} / t_{0}$, all other quantities by the references defined above and $\rho_{0} q_{1}^{0}=4 / 3 \pi \rho_{1} r_{0}^{3} n_{0}$ is the liquid water density for $n_{0}$ particles per unit volume, all of radius $r_{0} . \eta(r)$ is the droplet nucleation function as defined in Eq. (3).

The calculation of the condensation and nucleation terms requires application of the laws that describe such processes at the microscopic level. Equally, the calculation of the liquid drift (given by Eq. (11)) requires knowledge of the physical laws that govern microscopic droplet motion. These microscopic laws are connected to the bulk equations via the probability distribution function, $f(r)$. These are the topics of this section.

\subsection{Condensation and evaporation}

The condensation/evaporation from a single droplet is calculated assuming diffusive transfer of vapour and heat from the droplet to the surrounding air. The diffusive equations are coupled to the energy conservation and Claussius-Claperion equations (Rogers and Yau, 1989), resulting in the following expression for the condensation rate:

$$
\frac{\mathrm{d} r}{\mathrm{~d} t}=\gamma \frac{q_{\mathrm{v}} / q_{\mathrm{v}}^{\mathrm{sat}}-1}{r},
$$

where $\gamma$ is a function of the pressure and temperature and $q_{\mathrm{v}}^{\text {sat }}$ is the fraction of vapour at the saturation pressure. Since the $\gamma$ dependence on the temperature is quite mild (Rogers and Yau, 1989), it is common practice to assume $\gamma$ as constant. In clouds, typically $\gamma \sim 10^{-10} \mathrm{~m}^{2} \mathrm{~s}^{-1}$. Using this expression, the evaporation/condensation term of Eq. (19) in non-dimensional units is

$$
\overline{r^{2} \frac{\mathrm{d} r}{\mathrm{~d} t}}=D a\left(q_{\mathrm{v}} / q_{\mathrm{v}}^{\mathrm{sat}}-1\right) \bar{r}
$$

where the non-dimensional number

$$
D a=\gamma t_{0} / r_{0}^{2}
$$

describes the ratio of the typical time of the flow, $t_{0}=L_{0} / U_{0}$, to the condensation/evaporation time, $r_{0}^{2} / \gamma$. This number is called the Damköhler number due to the analogy with reactive flows (Shaw, 2003). A high Damköhler number indicates a very fast change of phase, suggesting that the thermodynamic equilibrium approximation should be used. On the other hand, low Damköhler numbers suggests use of a more complex formulation than the equilibrium assumption. Using Eq. (22) in Eq. (19), the condensation/evaporation term reads

$$
C=3 q_{1}^{0} n D a\left(q_{\mathrm{v}} / q_{\mathrm{v}}^{\mathrm{sat}}-1\right) \bar{r}
$$

This expression is equivalent to the condensation function used in Lagrangian calculations (e.g. Kumar et al., 2013), with the difference that Lagrangian models typically account for variability in the vapour field within the grid volume $\left(C_{\mathrm{lag}} \propto \overline{\left(q_{\mathrm{v}} / q_{\mathrm{v}}^{\text {sat }}-1\right) r}\right)$.

\subsection{Inertia and settling}

The equation of motion for a single droplet in a cloud is written in the Stokes limit (Maxey and Riley, 1983):

$$
\mathrm{d} \mathbf{v}_{\mathrm{r}}^{\mathrm{D}} / \mathrm{d} t=\left(1 / \tau_{\mathrm{d}}\right)\left(\mathbf{u}-\mathbf{v}_{\mathrm{r}}^{\mathrm{D}}\right)+\mathbf{g},
$$

where $\tau_{\mathrm{d}}=2 \pi r^{2} \rho_{\mathrm{l}} /\left(9 \mu_{\mathrm{g}}\right)$ is the particle response time and $\mu_{\mathrm{g}}$ is the viscosity of the surrounding air. For typical cloud temperatures, droplets of $10 \mu \mathrm{m}$ diameter have a typical response time $\tau_{\mathrm{d}} \sim 2.8 \times 10^{-4} \mathrm{~s}$. Equation (25) has a simplified solution when $\tau_{\mathrm{d}}$ is small compared with the typical time of the flow. The simplified solution in non-dimensional form reads

$$
\mathbf{v}_{\mathrm{r}}^{\mathrm{d}}=\mathbf{u}+S v_{\mathrm{r}} \mathbf{e}_{\mathrm{g}}-S t_{\mathrm{r}} \mathrm{d} \mathbf{u} / \mathrm{d} t+\mathcal{O}\left(S t^{2}\right),
$$

where $\mathbf{e}_{\mathrm{g}}$ is the unit vector in the direction of gravity. This equation defines two non-dimensional numbers. The settling number

$$
S v_{\mathrm{r}}=g \tau_{\mathrm{d}} / U_{0}
$$

is the ratio of the sedimentation velocity, $u_{\mathrm{s}}=g \tau_{\mathrm{d}}$, to the typical flow velocity scale and the Stokes number

$$
S t_{\mathrm{r}}=\tau_{\mathrm{d}} / t_{0}
$$

is the ratio between the particle response time and the typical time of the flow. In Eq. (26), it is easy to see how small droplets, with correspondingly small settling and Stokes number, follow the trajectory of the air, whereas bigger particles can escape these trajectories, which in turn translates into a drift flux. Boffetta et al. (2007) showed that the Eulerian treatment of this drift flux (as in this article) agrees with fully Lagrangian calculations in the limit $S t<1$ (defining the Stokes number with the Kolmogorov time scale).

The mean inertial drift velocity, $\mathbf{d}$, which appears in the Eulerian transport equations (Eq. (13)), is calculated from Eqs (11) and (26), neglecting terms of $\mathcal{O}\left(S t^{2}\right)$. In non-dimensional units, it reads

$$
\rho q_{1} \mathbf{d}=q_{1}^{0} n\left(S v \mathbf{e}_{\mathrm{g}}-S t \mathrm{~d} \mathbf{u} / \mathrm{d} t\right) \overline{r^{5}},
$$

which depends on the fifth moment of the droplet radius probability density function. The non-dimensional numbers $S v$ and $S t$ in Eq. (29) are defined for reference radius $r_{0}$.

\subsection{Nucleation and the equation for number density}

The calculation of the condensation and drift terms in Eqs (24) and (29) requires the number density $n$. The non-dimensional transport equation for the number density is calculated by integrating Eq. (3) and using the expression for the drift velocities given by Eq. (10):

$$
\begin{aligned}
& \frac{\partial n}{\partial t}+\nabla \cdot(n \mathbf{u})=-\nabla \cdot \mathbf{j}_{n}+\int \eta(r) \mathrm{d} r, \\
& -\mathbf{j}_{n}=n \overline{\mathbf{v}_{\mathrm{r}}-\mathbf{V}_{\mathrm{g}}}-n q_{\mathrm{l}}\left(\mathbf{V}_{\mathrm{l}}-\mathbf{V}_{\mathrm{g}}\right),
\end{aligned}
$$

where the drift flux consists of two different drift velocities, one weighted by the mass of the particles and another one weighted by the number of particles.

The number density has a clear definition inside the cloud, but there is some ambiguity about its definition in dry air. Some authors (Andrejczuk et al., 2004) assume that $n$ only represents droplets and therefore $n$ vanishes outside the cloud. Droplets are destroyed when they exit the cloud, while new droplets are nucleated for some given thermodynamical conditions. In this case, the nucleation or destruction of droplets in the cloud has to be modelled by the nucleation function $\eta(r)$. 
An alternative view (for example Heus et al., 2010) considers that $n$ represents the number of droplets plus the number of aerosols able to nucleate droplets once the vapour pressure surpasses the equilibrium level. This means that the dry air includes droplets of zero radius that do not carry any liquid water. One advantage of this approach becomes apparent in the case in which aerosols cannot be nucleated or destroyed because the nucleation term vanishes:

$$
\eta(r)=N=0 .
$$

The formulation developed in this article is valid for both interpretations of $n$, which differ only in the initial and boundary conditions for $n$ and the form of the nucleation term. Due to its simplicity, we decided to use the approach in which $n$ is the number of droplets plus aerosols and to neglect the nucleation term.

The droplets-plus-aerosol view also presents a clear advantage in the case in which droplets and aerosols are well mixed, so that $n$ does not present large variations around its mean $\bar{n}$. In this case, only the inertial drift in Eq. (30) is able to change the initial state, creating preferential concentrations. In clouds this drift is very slow compared with other typical cloud processes and therefore it will take relatively long to create appreciable deviations from $\bar{n}$. In this case, it is a good approximation to consider $n=\bar{n}$ so that Eq. (30) does not need to be solved. Another justification for this approximation is that $n$ only appears in the inertial drift and condensation terms of the transport equations (Eqs (11) and (19)). These terms have been presented as corrections to the original bulk equations. The small variations around $\bar{n}$ then appear as second-order corrections in the bulk equations and are therefore negligible.

\subsection{The log-normal probability distribution function}

The probability density function $f$ can generally be calculated by solving Eq. (3) (Williams, 1985). However, this operation is computationally very costly because Eq. (3) has to be solved in a five-dimensional space $\{\mathbf{x}, r, t\}$ (see for example Pantano and Shotorban, 2007). On the other hand, in DNS of clouds it is also desirable to increase the Reynolds numbers (or the size of the domain) as much as possible, which is also computationally very expensive. A good compromise is to assume a given form of the pdf.

In this article, we consider only single-mode probability distribution functions, as observed in warm clouds with no rain formation (Shaw, 2003). In particular, we focus on the lognormal distribution, which is commonly used to describe small droplets in clouds, due to its simplicity and ability to reproduce observations (Pruppacher and Klett, 1997). The general form of this distribution reads

$$
f(r)=\frac{n}{r \sigma \sqrt{2 \pi}} \exp \left(\frac{-(\ln r-\mu)^{2}}{2 \sigma^{2}}\right),
$$

where $\mu$ is a parameter related to the mean radius and $\sigma$ is related to the broadening of the distribution. In the atmospheric context, it is common to use the geometric standard deviation, $\sigma_{\mathrm{gc}}=\exp (\sigma)$, instead of $\sigma$. In LES of clouds, $\sigma_{\mathrm{gc}}$ is assumed to be constant for the whole simulation domain and its value is estimated from field measurements. Typical values of $\sigma_{\mathrm{gc}}$ range from 1.2 to 1.5 (Bretherton et al., 2007; Ackerman et al., 2009). It is, however, arguable that the same values of $\sigma_{\mathrm{gc}}$ are applicable to a DNS simulation. Field measurements typically provide averages over large domains, which enclose different regions with large and small droplets. In a DNS we aim to resolve those smaller regions which, if measured independently, should show narrower pdfs. Therefore, we think that the appropriate value of $\sigma_{\mathrm{gc}}$ for a DNS is below the LES value $\left(\sigma_{\mathrm{gc}}^{\mathrm{DNS}}<1.2\right)$, probably close to the Dirac delta distribution limit $\left(\sigma_{\mathrm{gc}}=1\right)$. The exact determination of $\sigma_{\mathrm{gc}}$ is another aspect of the problem that would benefit from more detailed field measurements or Lagrangian studies.

One advantage of the log-normal distribution is that the moments of the distribution can be calculated analytically:

$$
\overline{r^{s}}=\exp \left(s \mu+s^{2} \sigma^{2} / 2\right) .
$$

Using this equation, we calculate the expressions for the drift velocity, condensation/evaporation and flux term for the droplets number density:

$$
\begin{aligned}
\rho q_{\mathrm{l}} \mathbf{d}= & \left(\rho q_{\mathrm{l}}\right)^{5 / 3}\left(n q_{\mathrm{l}}^{0}\right)^{-2 / 3} \exp \left(5 \sigma^{2}\right)\left(S v \mathbf{e}_{\mathrm{g}}-S t \mathrm{~d} \mathbf{u} / \mathrm{d} t\right) \\
C= & 3 D a \exp \left(\sigma^{2}\right)\left(q_{\mathrm{v}} / q_{\mathrm{v}}^{\mathrm{sat}}-1\right)\left(\rho q_{1}\right)^{1 / 3}\left(q_{1}^{0} n\right)^{2 / 3} \\
& -\mathbf{j}_{n}=\frac{\mu}{R e S c_{1}} \nabla n-n\left[\exp \left(-9 \sigma^{2} / 2\right)-q_{1}\right] \mathbf{d}
\end{aligned}
$$

where all quantities have been normalized as described above. In Eq. (36), the diffusion term $\left(\mu /\left(R e S c_{1}\right) \nabla n\right)$ is a simplification, under the assumptions that $q_{1} / S c_{1} \ll 1$ and that the liquid-phase diffusion coefficient based on the number density is equal to the liquid-phase diffusion coefficient based on the mass density $\kappa_{1}$. The main reason to write this approximation is that the droplet diffusion term is in principle negligible in the limit $S c_{1} \rightarrow \infty$ and its main function is to avoid strong gradients in the $q_{1}$ and $n$ fields. We emphasize again that the properties of interest in the solutions of the equations should be independent of the liquidphase diffusion and extra simulations should be performed in order to test how the results vary for different values of $S c_{1}$.

The broadening of the pdf, $\sigma$, appears only in an exponential factor that multiplies the Stokes, settling and Damköhler numbers in the drift and condensation terms (Eqs (34) and (35)). For a narrow distribution $\left(\sigma_{\mathrm{gc}}=1.2\right)$, the Stokes and settling numbers increase by $18 \%$ and the Damköhler number increases by only $3 \%$ when compared with the Dirac distribution $\left(\sigma_{\mathrm{gc}}=1\right)$. In the broader distribution $\left(\sigma_{\mathrm{gc}}=1.5\right)$, the Stokes and settling numbers increase by $220 \%$ whereas the Damköhler number is increased by $18 \%$, again when compared with the Dirac distribution. The non-diffusional drift and settling are therefore much more sensitive to the broadening of the distribution than the condensation/evaporation term. Note also that the drift in the number density equation (36) is a factor $\exp \left(-9 \sigma^{2} / 2\right)$ smaller than the drift in the liquid equation (34). This difference is due to the largest droplets. These droplets feel stronger inertia and carry more liquid than average, so that their contribution is larger in the liquid equation than in the droplet density equation.

\section{Evaporatively driven cloud-top mixing layer}

\subsection{The buoyancy reversal instability}

We apply the above presented formulation for study of the buoyancy reversal instability (BRI) in stratocumulus clouds. At the stratocumulus top, a turbulent mixing layer forms between the cloud (cool and moist) and the free atmosphere on top (warm and dry). The BRI is triggered by evaporative cooling when some proportions of the mixture of dry and cloudy air are colder than the cloud bulk, as shown in Figure 1. As those parcels fall into the cloud bulk, they induce a turbulent motion that enhances the turbulent mixing at the cloud top and feeds back the instability. In the last years, it has been long debated to what extent the BRI is able to promote a large-scale flow in stratocumuli (Wood, 2012) and some authors have proposed that the BRI is crucial for cloudtop entrainment instability (CTEI), which leads to cloud breaking (Randall, 1980; Deardorff, 1980). However, measurements in the DYCOMS-II field campaign (Faloona et al., 2005) did not show any correlation between cloud mixing and the strength of the BRI and tank experiments suggest that the strength of the instability in stratocumuli is too weak to break the cloud interface (Shy and Breidenthal, 1990; Siems and Bretherton, 1992). 


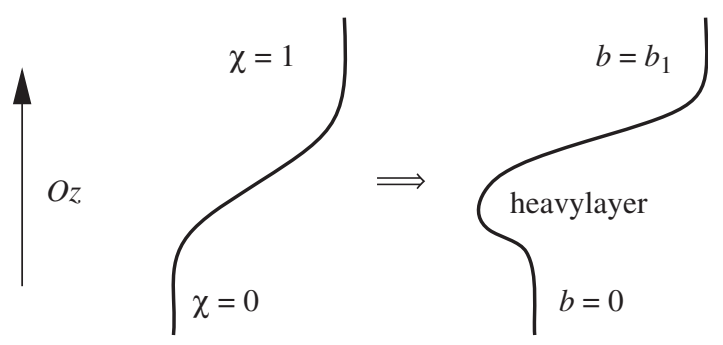

Figure 1. Vertical structure of the cloud top in terms of the mixture fraction $\chi$ and buoyancy $b$. The condition for the buoyancy reversal instability is that mixtures characterized by intermediate values of $\chi$ are less buoyant than the cloud bulk.

The strength of the BRI is typically measured by the nondimensional ratio $D=-b_{s} / b_{1}$ between the saturation buoyancy $b_{\mathrm{s}}$ and the buoyancy difference across the inversion $b_{1}$. The saturation buoyancy $b_{\mathrm{s}}$ is the minimum buoyancy that results from linearly mixing cloudy air with dry air. Typical values of $D$ in stratocumuli are modest, around $D=0.03$. However, the positive feedback in the BRI makes it difficult to predict the importance of this instability, even at such small values of $D$.

Mellado et al. (2009) and Mellado (2010) investigated the BRI in a mixing-layer configuration theoretically and numerically, using DNS with the equilibrium formulation. These studies were focused on the role of small-scale turbulence but neglected radiation and all small-scale processes resulting from the droplet dynamics, except for latent heat effects. The main findings of those investigations were as follows: (1) the BRI indeed leads to turbulence and enhances the mixing at the cloud top, but this turbulence is much weaker than that observed in clouds and the induced mixing is not strong enough to break the cloud deck; and (2) the typical length-scales characteristic of the mixing and the BRI are small, of the order of centimetres. The first conclusion is in accordance with the experiments and measurements cited above, suggesting that the BRI is not a main source of turbulence in stratocumulus clouds. The second conclusion suggests that the problem initially posed might not capture all aspects of the mixing, because the neglected small-scale processes might play an important role. However, all smallscale processes (except maybe for preferential concentration) are expected to work against the BRI (Mellado et al., 2010a). It is therefore expected that those equilibrium simulations provide a solid upper bound for the turbulent intensity generated by the BRI alone. This is in agreement with Bretherton et al. (2007), who showed a $7 \%$ reduction in entrainment by adding sedimentation to a stratocumulus LES.

In this section we assess quantitatively the role of the small-scale processes for BRI in a cloud-top mixing layer. We investigate the basic configuration presented in Mellado et al. (2010b) but use the extended formulation described in the previous section. In particular, we focus on finite-rate phase change, sedimentation, inertial effects and low liquid-phase diffusion.

\subsection{Set-up}

The cloud-top mixing layer consists of two infinite (unbounded) horizontal layers: one layer of warm and unsaturated fluid on top of a second layer of cooler and saturated (condensate laden) fluid, the gravity force acting downward. A sketch of such a mixing layer is shown in Figure 1.

Our investigation aims to provide an upper limit for the turbulent intensity solely generated by the BRI. The properties of the mixed layer are chosen to mimic the cloud-top properties measured in the reference flight RF01 of the DYCOMSII campaign (Stevens et al., 2003), but with an increased buoyancy reversal parameter. As measured in that flight, the freeatmosphere top layer has a vapour concentration $q_{\mathrm{t}}^{\mathrm{d}}=1.5 \mathrm{~g} \mathrm{~kg}^{-1}$ and a temperature $T^{\mathrm{d}}=19.1^{\circ} \mathrm{C}$ at a pressure $p=940 \mathrm{hPa}$. The properties of the cloud bottom layer have been adjusted to increase the buoyancy reversal parameter to $D=0.133$, four times larger than found in the cited flight: total water $q_{\mathrm{t}}^{\mathrm{c}}=12 \mathrm{~g} \mathrm{~kg}^{-1}$ and temperature $T^{\mathrm{c}}=11.3^{\circ} \mathrm{C}$, instead of the measured $q_{\mathrm{t}}^{\mathrm{c}}=$ $9 \mathrm{~g} \mathrm{~kg}^{-1}$ and $T^{\mathrm{c}}=10.6^{\circ} \mathrm{C}$. In thermodynamic equilibrium, this corresponds to a liquid water concentration $q_{1}^{\mathrm{c}}=3.1 \mathrm{~g} \mathrm{~kg}^{-1}$, well above the typical concentrations in stratocumuli. For simplicity, we set the Prandtl number equal to $1, \operatorname{Pr}=1.0$, which is close enough to the atmospheric value. We also define the reference values $C_{\mathrm{p}, 0}=1.007 \mathrm{~kJ} \mathrm{~kg}^{-1} \mathrm{~K}^{-1}$ and $T_{0}=300 \mathrm{~K}$.

The microphysical properties at the cloud bulk are calculated for droplets of diameter $10 \mu \mathrm{m}$, as typical of stratocumi (Stevens et al., 2003). Following Rogers and Yau (1989), the condensation/evaporation time is $r_{0}^{2} / \gamma=1 \mathrm{~s}$, the particle response time is $\tau_{\mathrm{d}}=2.8 \times 10^{-4} \mathrm{~s}$ and the settling velocity is $u_{\mathrm{s}}=2.74 \mathrm{~mm} \mathrm{~s}^{-1}$. When studying differential diffusion, we investigate two cases in which the liquid-phase diffusion is reduced by factors of five and ten. The corresponding Schmidt numbers $S c_{1}=5,10$ are still far from atmospheric values $\left(S c_{1} \sim 10^{7}\right)$, but the main purpose of these simulations is to evaluate the impact of a reduced liquid-phase diffusion on the flow.

Mellado (2010) showed that a relatively small simulation domain ( $L \sim 3 \mathrm{~m}$ in the vertical) is enough to capture buoyancy reversal effects on the turbulence. In such a small vertical domain, the Boussinesq formulation provides a valid approximation which greatly simplifies the evolution equations (13). Using this approximation and substituting the equation for the energy $e$ with the equation for the enthalpy $h$, we obtain

$$
\begin{aligned}
& \nabla \cdot \mathbf{u}=0 \\
& \partial / \partial t(\mathbf{u})+\mathbf{u} \cdot \nabla \mathbf{u}=-\nabla p+\frac{1}{R e} \nabla^{2} \mathbf{u}+R i \frac{\rho-\rho_{0}}{\Delta \rho} \mathbf{e}_{\mathrm{g}}, \\
& \partial / \partial t\left(q_{\mathrm{t}}\right)+\mathbf{u} \cdot \nabla q_{\mathrm{t}}=-\nabla \cdot \mathbf{j}_{\mathrm{t}} \\
& \partial / \partial t\left(q_{1}\right)+\mathbf{u} \cdot \nabla q_{1}=-\nabla \cdot \mathbf{j}_{1}+C \\
& \partial / \partial t(h)+\mathbf{u} \cdot \nabla h=-\nabla \cdot \mathbf{j}_{\mathrm{q}},
\end{aligned}
$$

with the flux terms given by Eq. (14). In the non-equilibrium cases we assume the aerosols-plus-droplets interpretation and that the number density $n$ is homogeneous and constant. In this approximation, the equation for the number density does not need to be solved. In section 3.3, we saw that this approximation holds when the pure inertial forces and sedimentation are small. We also assume that the droplet radius probability distribution function is a Dirac function $\left(\sigma_{\mathrm{gc}}=1\right)$. Under these conditions, the condensation/evaporation term $C$ and inertial drift $\mathbf{d}$ are given by Eqs (35) and (34), respectively. The equation for liquid water in Eq. (37) is used just in the cases with finite Damköhler number. In cases of infinitely fast change of phase, we calculated $q_{1}$ from $h$ and $q_{\mathrm{t}}$ using the equilibrium concentrations, as explained in Mellado et al. (2010b).

We use the DNS code previously described in Mellado (2010), which has been modified to include the new formulation. The physical horizontal size of the domain is $L_{x} \times L_{y}=3 \times 3 \mathrm{~m}^{2}$. All simulations use a cubic grid with $1024^{3}$ points. The grid was stretched in the vertical dimension to separate the region where the flow develops from the top and bottom boundaries, minimizing the effect of the boundary conditions. The grid is isotropic in the region where the flow develops, with spacing $\mathrm{d} x=\mathrm{d} y=\mathrm{d} z=2.9 \mathrm{~mm}$. The time step has been adjusted using a Courant constraint and it is of the order of $\mathrm{d} t=20 \mathrm{~ms}$ for the equilibrium case. In the cases of finite-time evaporation, an even shorter time step was employed to resolve the thermodynamic change of phase ( $\mathrm{d} t=8.5 \mathrm{~ms})$. In those cases, we also set to zero the negative liquid water concentrations (of order $q_{1} \sim 5 \times 10^{-5}$ ) that might arise due to numerical error before each time step. Otherwise the condensation/evaporation term, $C$ in Eq. (35), is not properly defined. Sensitivity studies have been performed to ascertain that this clipping does not affect the results discussed here. 


\subsection{Initialization using the equilibrium formulation}

When including sedimentation in the non-equilibrium formulation, the problem becomes very sensitive to the initial conditions. If the initial turbulence is too weak then the sedimentation has enough time to evacuate the droplets from the inversion before any appreciable mixing occurs. The BRI, and therefore any source of turbulence, is suppressed and the flow becomes laminar. If the turbulence at the initial condition is too strong, then the spin-up period can be very long, of the order of the integral time-scale. It is then difficult to distinguish which flow properties are just an effect of the initial condition. In this article, the initial conditions are generated from a simulation with the equilibrium formulation under the assumption that the flow dynamics are similar in the equilibrium and non-equilibrium cases.

We start with a simulation of the mixed layer described in the previous section using the equilibrium formulation, therefore neglecting all small-scale processes. The flow in this simulation evolves as described in Mellado (2010): after a short transient, a self-preserving convective boundary layer $(\mathrm{CBL})$ grows into the cloud bulk, while mixing at the cloud top is described by a constant entrainment velocity. In this simulation there is no sedimentation and therefore the initial conditions are quickly forgotten. We stop the simulation after the CBL reaches the self-preserving state, when the CBL fills one third of the horizontal domain size. The last stage of this equilibrium simulation is the reference case, which is used as initial condition for all the simulations presented in the next section.

The reference case corresponds to a CBL of depth $2 z^{*}=1 \mathrm{~m}$ with a turbulent dissipation rate $\epsilon 0=5 \times 10^{-5} \mathrm{~m}^{2} \mathrm{~s}^{-3}$. The cloud-top inversion is characterized by an entrainment velocity $w_{\mathrm{e}}=\mathrm{d} z_{\mathrm{i}} / \mathrm{d} t=1.12 \mathrm{~mm} \mathrm{~s}^{-1}$ and by an inversion thickness $\delta_{\mathrm{i}}=\Delta q_{\mathrm{t}} /\left\langle\partial q_{\mathrm{t}} / \partial z\right\rangle_{z_{\mathrm{i}}}=42 \mathrm{~mm}$. These quantities define the typical inversion time $t_{\mathrm{i}}=\delta_{\mathrm{i}} / w_{\mathrm{e}}=37.5 \mathrm{~s}$. The inversion point, $z_{\mathrm{i}}$, is here defined as the height of the maximum gradient of the horizontally averaged total water profile. The CBL is characterized by a convective Reynolds $R e^{*}=w^{*} z^{*} / v \simeq 10^{3}$ and by a convective Richardson number $R i^{*}=b_{1} z^{*} /\left(w^{*}\right)^{2} \simeq 150$, where $w^{*}$ is an integral velocity scale. The convective scales $z^{*}$ and $w^{*}$ were defined using the expressions presented in Mellado (2010). Due to the small size of the domain, the convective Reynolds number is much lower than typical values in clouds. However, the Richardson number and dissipation rate are still in the range that can be found in stratocumuli (Vaillancourt and Yau, 2000). We note that the dissipation rate and inversion thickness are independent of the size of the CBL and therefore these would be the same if we run the equilibrium simulation much longer. This is important because those scales determine the smallest flow scales that are supposed to interact the most with the droplet dynamics, once the non-equilibrium formulation is included. Therefore the interaction of the droplet dynamics with the flow in our simulations should be similar to that found in larger domains, except for the effect of turbulent internal intermittency, which increases with $R e^{*}$ (Shaw, 2003).

Before discussing the non-equilibrium simulations, we estimate which small-scale features are relevant for the cloudtop dynamics by looking at the non-dimensional numbers that relate the flow properties of the reference case to the microphysical properties. The turbulent flow length-scales in the CBL span several orders of magnitude, from the Komogorov scale, $\eta=\left(v^{3} / \epsilon\right)^{1 / 4}$, to the integral scale, commensurable with $z^{*}$. In principle the whole range of non-dimensional numbers bounded by those scales has to be considered. In addition, the cloud-top mixing introduces new velocity and length-scales that are relevant to the entrainment process. For a strongly stratified case, those scales are identified as the inversion thickness and the entrainment velocity (Mellado, 2010) and they are of diffusive nature. In Table 1, we present the typical integral, Kolmogorov and diffusion scales for the reference case. Using these three different scales, we can construct three different Damkhöler, settling and
Table 1. Scales and non-dimensional numbers that describe the problem in the reference equilibrium case (the initial condition for the other non-equilibrium simulations). The two first rows indicate the time- and length-scales corresponding to the integral, Kolmogorov and diffusive scales. The following rows show the Damköhler, settling and Stokes numbers based on each of those scales. These non-dimensional numbers were calculated using Eqs (23), (27) and (28) for droplets of 10 m diameter.

\begin{tabular}{lccc}
\hline & Integral & Kolmogorov & Diffusion \\
\hline Time & $17.1 \mathrm{~s}$ & $0.45 \mathrm{~s}$ & $37.5 \mathrm{~s}$ \\
Length & $490 \mathrm{~mm}$ & $2.7 \mathrm{~mm}$ & $42 \mathrm{~mm}$ \\
$\mathrm{Da}$ & 17.1 & 0.45 & 37.5 \\
Sv & 0.096 & 0.46 & 2.4 \\
St & $1.7 \times 10^{-5}$ & $6.4 \times 10^{-4}$ & $7.7 \times 10^{-6}$ \\
\hline
\end{tabular}

Stokes numbers as presented in Table 1. The liquid Schmidt number is not shown in the table because it is independent of the flow properties.

All Stokes numbers are much smaller than one, indicating that pure inertial effects should not be significant. The Damköhler number is close to one only for lengths comparable to the Kolmogorov scale, so that we expect to see variations due to finite-time thermodynamics only at the smallest scales of the flow. The settling parameter is close to one for the Kolmogorov scale and larger than two for the diffusion scale. We can then expect that the settling will play an important role for the mixing process across the inversion.

It is worth noting that the size of the domain achieved in this simulation $(1 \mathrm{~m})$ is large enough to neglect all microphysical effects based on the integral scale, as indicated by the nondimensional numbers in Table 1. Small-scale effects are therefore not expected to interact directly with the large-scale flow, which serves as a justification for the small size of the simulations. This does not mean that small-scale processes are negligible. Small-scale processes can indirectly alter the large-scale flow by modifying mixing at the inversion, because the amount of dry air mixed into the cloud determines the buoyancy flux into the CBL. The non-dimensional numbers indicate that the inversion dynamics, which depends on molecular diffusion and small eddies, might be modified by small-scale processes and therefore the indirect impact of small-scale features on the flow dynamics cannot be neglected.

\subsection{Dynamics using the non-equilibrium formulation}

We explore the effect of the small-scale processes on the BRI using seven simulations (see Table 2). All simulations start from the reference case described in the previous section and differ in which small-scale processes are active. In brief: we perform one simulation in which we continue with the equilibrium formulation, five simulations in which sedimentation, differential diffusion (two cases), supersaturation and pure inertial effects are independently included and one simulation that combines all the small-scale processes described above.

Table 2. This table indicates which small-scale features have been included in each simulation and the Schmidt number associated with the liquid-phase diffusion. 'No' indicates that the equilibrium formulation was used for this particular feature. 'Yes' means that the small-scale features were modelled for $10 \mathrm{~m}$ diameter droplets, as described in the text.

\begin{tabular}{lccrc}
\hline$\#$ & Sedimentation & Inertia & $S c_{1}$ & Finite-rate therm. \\
\hline I & No & No & 1 & No \\
II & No & Yes & 1 & No \\
III & No & No & 1 & Yes \\
IV & No & No & 5 & No \\
V & No & No & 10 & No \\
VI & Yes & No & 1 & No \\
VII & Yes & Yes & 10 & Yes \\
\hline
\end{tabular}




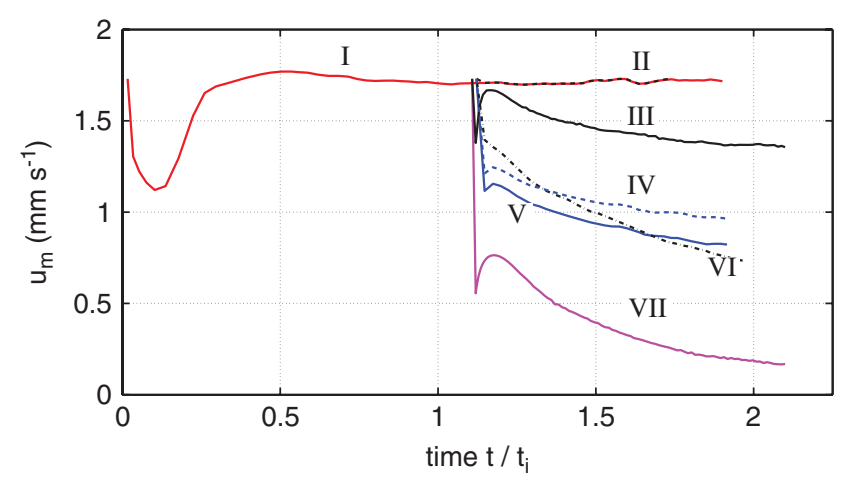

Figure 2. Rate of liquid water mixed out of the cloud into the dry atmosphere The top line (red in the online article) corresponds to a simulation using the equilibrium formulation (case I). The state at $t_{\mathrm{i}}=1.1$ in this simulation is used as the initial condition for all other simulations. In these we investigate independently the small-scale features coming from the droplet dynamics using the non-equilibrium formulation. All simulations are summarized in Table 2. The black dashed line (case II) corresponds to pure inertia. The black solid line (case III) corresponds to finite-time phase change. The next lines (blue in the online article) correspond to reduced liquid-phase diffusion, the dashed line (case IV) for $S c_{1}=5$ and the solid line (case V) for $S c_{1}=10$. The black dash-dotted line (case VI) corresponds to settling. The bottom line (case VII, magenta in the online article) corresponds to a case in which all small-scale effects were introduced.

We compare the different cases by quantifying the rate at which cloud water evaporates in the mixing process. This quantity is calculated by integrating Eq. (13) in a control volume, the top and bottom boundaries of which are placed far away from the cloud top:

$$
u_{\mathrm{m}}=-\frac{1}{q_{\mathrm{l}}^{\mathrm{c}} S} \int C \mathrm{~d} \mathbf{x}=-\frac{1}{q_{\mathrm{l}}^{\mathrm{c}} S} \frac{\mathrm{d} \int q_{\mathrm{l}} \mathrm{d} \mathbf{x}}{\partial t}-u_{\mathrm{s}}\left(1-q_{\mathrm{l}}^{\mathrm{c}}\right),
$$

where $S$ is the horizontal cross-section of the control volume, $q_{1}^{c}$ is the cloud-bulk liquid water, $u_{\mathrm{s}}$ is the settling velocity and $u_{\mathrm{m}}$ is the integrated evaporation rate, scaled to be a positive velocity It is useful to scale the evaporation rate in this way because its magnitude is then comparable to the entrainment velocity. The exact relationship between both velocities is, however, nontrivial. Using $u_{\mathrm{m}}$ instead of the entrainment velocity has some advantages. First, $u_{\mathrm{m}}$ is proportional to the integrated evaporative cooling of the mixing layer, the only source of turbulence of the BRI. Second, its definition is independent of the inversion-point choice. This second property of $u_{\mathrm{m}}$ becomes very useful in the non-equilibrium formulation, when the different cloud species have their own dynamics, allowing for several definitions of the reference inversion point $z_{\mathrm{i}}$.

In Figure 2, we show the time evolution of $u_{\mathrm{m}}$. At early times only the case with the equilibrium formulation is shown (case I). Notice that this equilibrium case tends to an asymptotic state after a short transient $\left(t>0.5 t_{\mathrm{i}}\right)$. All other simulations start from this reference case at $t=1.1 t_{\mathrm{i}}$.

When only pure inertial effects are included (case II), there is no variation in the mixing with respect to the equilibrium case, as shown in Figure 2. This behaviour was anticipated from the estimate of small Stokes numbers in the previous section. Visual inspection shows short-lived regions with liquid water concentrations slightly higher than in the cloud bulk, but these are too small to have any impact on the flow dynamics. It has been proposed that high Reynolds number intermittency might enhance the inertial effects (Shaw, 2003), but the Reynolds numbers achieved here are too low to address this question.

Finite-time thermodynamics (case III) has a stronger impact on the mixing and reduces $u_{\mathrm{m}}$ by $25 \%$. We can explain this tendency qualitatively by looking at the inversion balance proposed by Mellado (2010) for the equilibrium formulation. This balance is based on a Rayleigh number analysis and as such it can be understood by looking at the competition of diffusion and buoyancy when a perturbation is introduced.
While molecular diffusion smooths out this perturbation, the destabilizing buoyancy generated by evaporative cooling drives the perturbation out of the inversion. According to this criterion, the inversion thickness adjusts so that the times required for both processes (diffusion and free fall) are comparable. When a finitetime change of phase is included both times have to be adjusted. While the diffusion time is independent of the condensation time (for $S c_{1}=1$ ), the time that the perturbation requires to destabilize the inversion increases with evaporation time. The reason is that the perturbation now needs some extra time to cool down before the buoyancy can be effective. When adding this extra evaporation time to the escape time in the Rayleigh number analysis, the resulting inversion thickness broadens and the mixing rate decreases.

Many bulk models of clouds include the implicit assumption that the liquid-phase diffusion is equal to the vapour diffusion $\left(S c_{1}=1\right)$. The accuracy of this assumption remains to be investigated when the diffusion is of turbulent nature (as in LES), but the assumption is probably too coarse when the liquidphase diffusion characterizes the thermal fluctuations of the droplets (as in our highly resolved DNS). We observe here that when more realistic values of the liquid diffusion are used the strength of the BRI reduces considerably. Our simulations predict a $40 \%$ reduction of the BRI when decreasing the liquid diffusion (increasing $S c_{1}$ ) by a factor of five (case IV) and a $50 \%$ reduction when decreasing the diffusion by a factor of ten (case V). From this tendency, we speculate that a further decrease of the liquid diffusion should not reduce the mixing much more beyond these values, although Lagrangian studies should provide a more definitive answer to this question.

The strongest reduction of the evaporation rate $u_{\mathrm{m}}$ (between $50 \%$ and $60 \%$ ) occurs when settling is included (case VI). This behaviour was anticipated by looking at the high settling numbers in Table 1 and a more physical explanation is revealed below. When all the small-scale processes are put together (case VII), the negative effects on the entrainment add in a nonlinear way and the mixing is reduced by $90 \%$ at the end of the simulation. At this stage, $u_{\mathrm{m}}$ still shows a negative tendency, suggesting that the BRI might be completely suppressed. If this limit is reached, all turbulence would eventually die out and the cloud would reach a state with just molecular mixing.

We explain the strong mixing reduction induced by the smallscale processes first by looking at flow visualizations. In Figure 3, we compare the total water field of the simulation with all smallscale processes (case VII) to the same field in the fully equilibrium case (case I). Notice that in the CBL the equilibrium simulation presents a more a diffusive field due to the lower $S c_{1}$ but otherwise the flow there looks quite similar. The most important differences arise in the inversion layer. In the case with all small-scale features, a new layer appears between the cloud bulk and the inversion (green in Figure 3). This intermediate layer contains no liquid water but has a vapour concentration similar to that inside the cloud bulk. We observe that the addition of sedimentation evacuates the droplets from this middle layer, while reducing the liquid-phase diffusion hinders droplet diffusion back into the middle layer.

The middle layer described above is conceptually similar to the entrainment interface layer (EIL) observed in clouds by Gerber et al. (2005) and Haman et al. (2007), in the sense that it provides a cloud-free smooth transition between cloud and free atmosphere. The main difference between the middle layer and the EIL is that the middle layer in our simulations is much thinner, of the order of centimetres, and that its composition is much closer to the cloud bulk. It remains to be explored in larger simulations, with other turbulent sources, whether the middle layer in our simulations eventually grows and mixes with the free atmosphere, evolving into a EIL as observed in clouds.

The emergence of the middle layer modifies the mixing behaviour between the dry air and the cloud bulk. In Figure 4(a), we show the joint probability density function (jpdf) of total water 
(a)

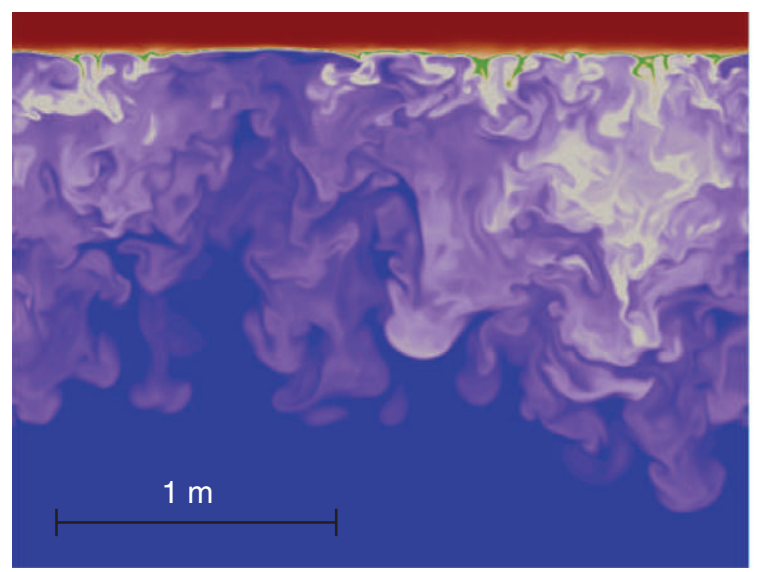

(b)

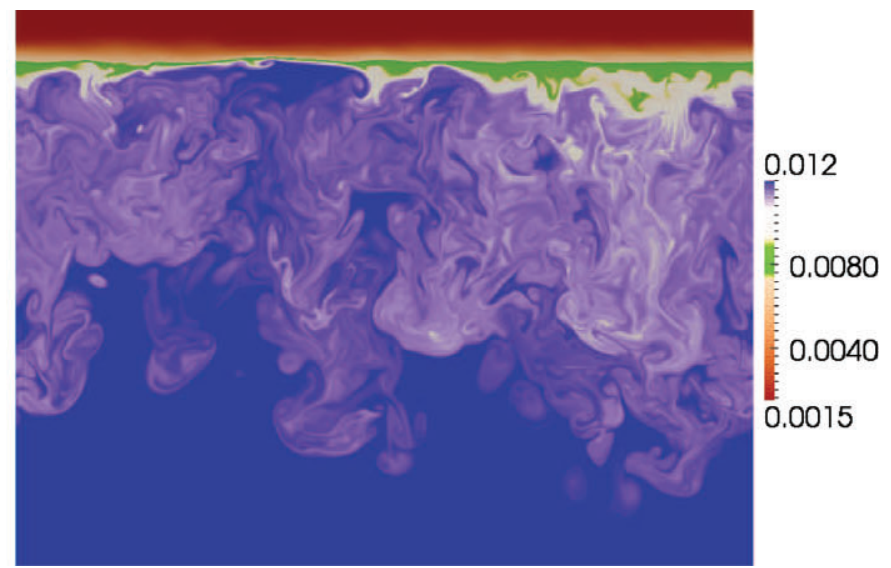

Figure 3. Total water cross-section at the final stage of the simulation for the cases with (a) pure equilibrium formulation $\left(t=1.9 t_{\mathrm{i}}\right)$ and (b) all small-scale processes included $\left(t=2.1 t_{\mathrm{i}}\right)$. The horizontal domain size is $3 \mathrm{~m}$. The colour scale varies from red, $q_{\mathrm{t}}=0.0015$, to blue, $q_{\mathrm{t}}=0.012$. The saturated vapour content in the cloud is $q_{\mathrm{v}}^{\text {sat }}\left(T^{\mathrm{c}}\right)=0.0089$. The green area indicates the area for total water where $0.85<q_{\mathrm{t}} / q_{\mathrm{v}}^{\text {sat }}\left(T^{\mathrm{c}}\right)<1$, which corresponds to regions empty of liquid water but still containing a large amount of vapour. Notice how in the case with all small-scale processes a layer depleted of liquid is formed between the cloud and the free atmosphere, while keeping the vapour interface (and also temperature) rather flat.
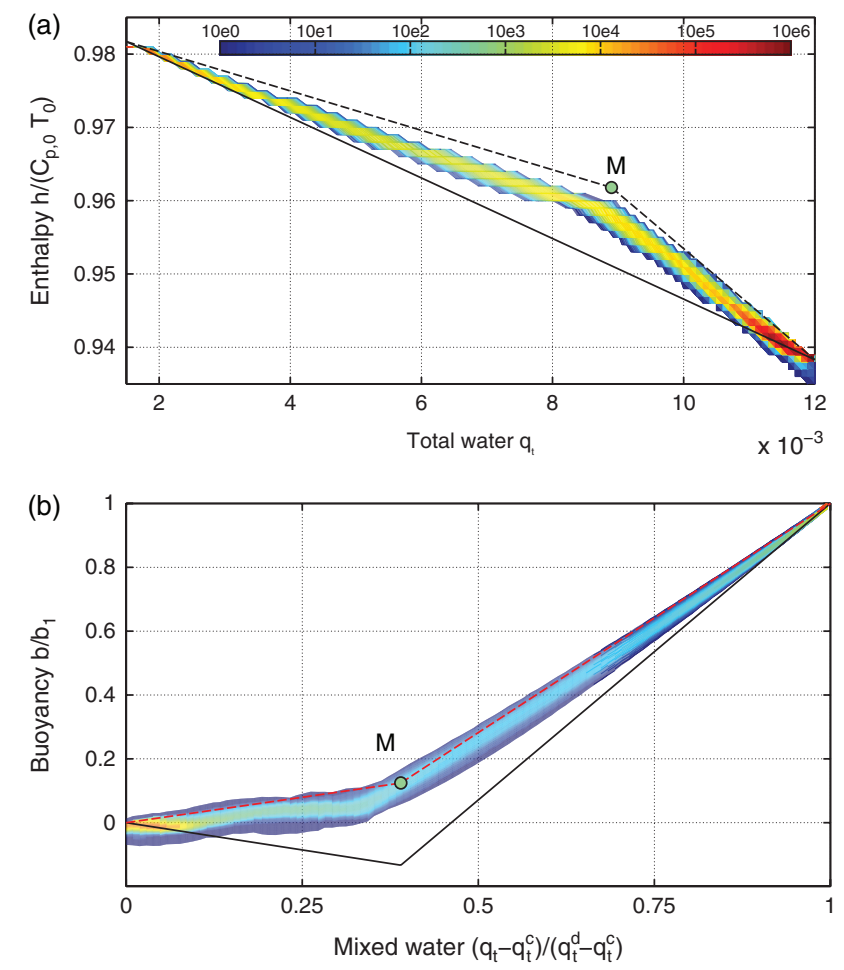

Figure 4. (a) Joint pdf of total water $q_{\mathrm{t}}$ and enthalpy $h /\left(C_{\mathrm{p}, 0} T_{0}\right)$. (b) Joint pdf of buoyancy $b / b_{1}$ and scaled total water $\left(q_{\mathrm{t}}-q_{\mathrm{t}}^{\mathrm{c}}\right) /\left(q_{\mathrm{t}}^{\mathrm{d}}-q_{\mathrm{t}}^{\mathrm{c}}\right)$. The scaled total water is equivalent to the mixed fraction in the equilibrium formulation. Both colour plots represent the last stage $\left(t=2.1 t_{\mathrm{i}}\right)$ of the simulation with all small-scale effects (case VII). The continuous line represents a linear mixing process. The point $\mathrm{M}$ corresponds to a middle layer with the same vapour and temperature as the cloud but no liquid water. The dashed line represents the mixing of the cloud and the free atmosphere with the middle layer.

and enthalpy for the simulation with all small-scale processes (case VII). Those results do not follow the linear mixing characteristic of the equilibrium formulation indicated by the solid line. In order to explain the observed jpdf, we introduce an idealized mixing process, the middle-layer mixing, similar to the EIL mixing described by Kurowski et al. (2009). We describe the middle-layer mixing as a process in which the cloudy air and the free atmosphere do not mix directly with each other and only mix with an idealized middle layer that is generated by droplet sedimentation. This middle layer contains saturated vapour at the cloud-bulk temperature but no liquid water and is represented by point $\mathrm{M}$ in Figure 4(a). For the middle-layer mixing, we also assume linear mixing between the middle layer and the cloud bulk and between the middle layer and the free atmosphere, as shown by the dashed line in Figure 4(a). The jpdf in Figure 4(a) falls in the interval between linear mixing and middle-layer mixing, but the latter seems to capture better the tendencies of the data.

The joint pdf of total water and buoyancy presented in Figure $4(\mathrm{~b})$ is also better described by middle-layer mixing compared with traditional linear mixing. Notice that there is no evaporation or condensation during middle-layer mixing: neither when the cloudy air mixes with the middle layer (all air is saturated) nor when the dry air mixes with the middle layer (no droplets involved). Therefore, middle layer mixing does not allow for the BRI. The buoyancy pdf in our simulations is pushed towards this process, explaining the strong reduction in BRI observed in Figure 2.

\section{Summary and conclusions}

We have developed a bulk formulation for direct numerical simulations of warm clouds that accounts for the small-scale features originating from the microscopic droplet dynamics: finite-time phase change, pure inertial effects and settling. These features are characterized by three non-dimensional parameters: a Damköhler number, a Stokes number and a settling parameter. This formulation is aimed at the study of mixing processes at cloud boundaries in domains of several metres. Those scales are too large for current Lagrangian models but are still poorly represented in current LES. We propose that bulk simulations using this or similar formulations could help to bridge this gap.

Our main approximations are the continuum description of the liquid droplets and the addition of artificially high diffusion to the liquid-phase dynamics. The addition of diffusion is necessary in order to relax the resolution constraints. The formulation provides a control parameter, the liquid Schmidt number $S c_{1}$, to ascertain the validity of this approximation.

The formulation has been developed under the assumption that the droplet radius pdf follows a log-normal distribution. The system is then reduced to the evolution equations for the velocities, total water mass fraction $q_{\mathrm{t}}$, internal energy $e$, liquidwater mass fraction $q_{1}$ and particle number density $n$. These equations include flux terms, which account for the relative movement of the droplets with respect to vapour and air. We investigate the limit in which the particles are well mixed, so that $n$ is approximately constant. In this well-mixed configuration, different log-normal distributions differ only by a multiplicative factor for the non-dimensional parameters introduced above. 
We apply the formulation to investigate buoyancy reversal instability (BRI) at the cloud top. With this purpose, we perform simulations of a mixed-layer configuration that mimics a cloud top driven solely by evaporative cooling. Pure inertia has a negligible effect, as predicted by the low Stokes numbers associated with the flow. All the remaining small-scale processes reduce the mixing rate. Settling and the reduction of the liquid-phase diffusion have the largest impact on the instability and decrease the mixing rate of liquid water between $50 \%$ and $60 \%$ with respect to the equilibrium formulation.

When all small-scale features are included, mixing with the free atmosphere is reduced by $90 \%$. We explain this strong reduction by looking at a middle layer that emerges between the cloud bulk and the inversion layer. This layer is generated when sedimentation evacuates the droplets from the cloud interface and therefore it contains no liquid water but has vapour concentrations and temperature similar to the cloud bulk. This middle layer is thus akin to the entrainment interface layer observed in clouds (Haman et al., 2007). When the middle layer grows, the cloud-bulk air and the dry air from the free atmosphere cannot mix directly; they have to mix previously with moist air from this middle layer. The mixing line and the joint pdf of total water and buoyancy are consistent with this picture. The mixing within the middle layer does not include any mixtures with buoyancy below the cloud-bulk buoyancy, suppressing the BRI.

In the previous investigation using the bulk formulation (Mellado, 2010), it was shown that the entrainment velocity is too low when the BRI is acting alone. We confirm here that the flow induced by the BRI gets much weaker when a better description of the droplet dynamics is included, as previously suggested in Mellado et al. (2010a). We conclude that the BRI and evaporative cooling can contribute significantly to the mixing only when other turbulent sources enhance mixing at the cloud top, e.g. radiation or shear. This is also in agreement with the experimental studies of Shy and Breidenthal (1990) and Siems and Bretherton (1992). In contrast to the low turbulence induced by evaporative cooling, we show in a different study (De Lozar and Mellado, 2013) that radiation alone can explain between $50 \%$ and $100 \%$ of the entrainment velocities measured in the DYCOMS-II campaign (Faloona et al., 2005). Radiation is thus probably the main source of turbulent energy in stratocumulus clouds, as originally proposed by Petterssen (1938) and generally supported by many other studies (see the review of Wood, 2012).

\section{A. Appendix: Summarized derivation of the transport equations}

In this Appendix we summarize the steps and approximations used to obtain the transport equations (Eq. (13)) from the drift velocities presented in Eq. (12). More details can be found in Mellado et al. (2010b).

The general form of the transport equations of property $A$ in a mixture composed of three species (in this case dry air, vapour and liquid water) is

$$
\partial\left(\sum_{i=\mathrm{v}, \mathrm{d}, \mathrm{l}} A_{i}\right) / \partial t+\nabla \cdot\left(\sum_{i=\mathrm{v}, \mathrm{d}, \mathrm{l}} \mathbf{V}_{i} A_{i}\right)=\sum_{i=\mathrm{v}, \mathrm{d}, \mathrm{l}} \xi_{i}
$$

where $A_{i}$ is the density of the quantity that carries each of the species, $\xi_{i}$ accounts for the source term and $\mathbf{V}_{i}$ is the centre-of-mass velocity of each species discussed in section 2.2. These conservation equations can be written in terms of the mean velocity $\mathbf{u}$ (defined in Eq. (6)) and the total density $A_{\mathrm{t}}=\sum_{i=\mathrm{v}, \mathrm{d}, \mathrm{l}} A_{i}$ as

$$
\begin{array}{r}
\partial A_{\mathrm{t}} / \partial t+\nabla \cdot\left(\mathbf{u} A_{\mathrm{t}}\right)=-\nabla \cdot \mathbf{j}_{\mathrm{D}}+\xi_{t}, \\
\mathbf{j}_{\mathrm{D}}=\sum_{i=\mathrm{v}, \mathrm{d}, \mathrm{l}} A_{i}\left(\mathbf{V}_{i}-\mathbf{u}\right),
\end{array}
$$

where the transport due to the movement of the different species with respect to the mean flow, the drift flux $\mathbf{j}_{D}$, appears explicitly. This drift flux is calculated using the drift velocities presented in Eq. (12).

As an example, we show the calculation of the transport equation for the total water $A_{\mathrm{t}}=\rho q_{\mathrm{t}}$. In this case, the mass density of vapour carried by the vapour is $A_{\mathrm{v}}=\rho q_{\mathrm{v}}$, the mass density of liquid carried by the liquid is $A_{1}=\rho q_{1}$ and the dry air carries no water, so that $A_{\mathrm{d}}=0$. Using Eq. (A2), the transport equation for the total water reads

$$
\begin{gathered}
\partial\left(\rho q_{\mathrm{t}}\right) / \partial t+\nabla \cdot\left(\rho \mathbf{u} q_{\mathrm{t}}\right)=-\nabla \cdot \mathbf{j}_{\mathrm{t}}, \\
\mathbf{j}_{\mathrm{t}}=\rho q_{\mathrm{l}}\left(\mathbf{V}_{\mathrm{l}}-\mathbf{u}\right)+\rho q_{\mathrm{v}}\left(\mathbf{V}_{\mathrm{v}}-\mathbf{u}\right),
\end{gathered}
$$

where we have used the condition that the total water is a conserved quantity in absence of solidification or precipitation $\left(\xi_{\mathrm{v}}+\xi_{1}=0\right)$. Using Eq. (12) for the drift velocities, the drift flux reads

$$
\mathbf{j}_{\mathrm{t}} / \rho=-\kappa_{\mathrm{v}} \nabla q_{\mathrm{t}}-\left(\kappa_{\mathrm{l}}-\kappa_{\mathrm{v}}\right) \nabla q_{\mathrm{l}}+\left(1-q_{\mathrm{t}}\right) q_{\mathrm{l}} \mathbf{D} .
$$

This equation is equivalent to the first line in Eq. (13), where the same expression is shown in a non-dimensional form. The rest of the transport equations are derived in the same way, but using total mass, total momentum, mass liquid and total energy. The main advantage of our choice of transport equations over other possible choices (such as using vapour or temperature) becomes apparent in the Boussinesq limit, where all production terms associated with compressibility are neglected in the internal energy equation, so that source terms only appear in the mass liquid equation.

The drift fluxes for the total mass, liquid and total water in Eq. (14) are exact. In the energy and momentum equations, we keep the leading-order terms that describe the droplet transport of momentum and mechanical energy and neglect the terms of order $q_{1}\left\|\mathbf{V}_{\mathrm{l}}-\mathbf{V}_{\mathrm{g}}\right\|$. It is easy to show that this approximation is valid in the limits $q_{1} S t \ll 1$ and $q_{1} S v \ll 1$, which are interesting for simulations of clouds. Notice that, by writing the formulation in terms of the mean velocity of all components (including the liquid), the leading-order terms of the transport are naturally incorporated into the equations and only higher orders terms enter into the drift flux.

\section{Acknowledgements}

Support from the Max Planck Society through its Max Planck Research Groups program is gratefully acknowledged. Computational time was provided by the Jülich Supercomputer Centre.

\section{References}

Ackerman AS, Kirkpatrick MP, Stevens DE, Toon OB. 2004. The impact of humidity above stratiform clouds on indirect aerosol climate forcing. Nature 432: 1014-1017. DOI: 10.1038/nature03174.

Ackerman AS, van Zanten MC, Stevens B, Savic-Jovic V, Bretherton CS, Chlond A, Golaz JC, Jiang H, Khairoutdinov M, Krueger SK, Lewellen D, Lock A Moeng C, Nakamura K, Petters M, Snider JR, Weinbrecht S, Zulauf M. 2009. Large-eddy simulations of a drizzling, stratocumulus-topped marine boundary layer. Mon. Weather Rev. 137: 1083.

Andrejczuk M, Grabowski WW, Malinowski SP, Smolarkiewicz PK. 2004. Numerical simulation of cloud-clear air interfacial mixing. J. Atmos. Sci. 61: $1725-1739$.

Andrejczuk M, Reisner JM, Henson B, Dubey MK, Jeffrey CA. 2008. The potential impacts of pollution on a nondrizzling stratus deck: Does aerosol number matter more than type? J. Geophys. Res. 113: D19 204. DOI: 10.1029/2007JD009445.

Andrejczuk M, Grabowski WW, Malinowski SP, Smolarkiewicz PK. 2009. Numerical simulation of cloud-clear air interfacial mixing: Homogeneous versus inhomogeneous mixing. J. Atmos. Sci. 66: 2493-2500. DOI: 10.1175/2009JAS2956.1.

Bodenschatz E, Malinowski SP, Shaw RA, Stratmann F. 2010. Can we understand clouds without turbulence? Science 327: 970-971. DOI: 10.1126/science. 1185138 . 
Boffetta G, Celani A, De Lillo F, Musacchio S. 2007. The Eulerian description of dilute collisionless suspension. EPL 78: 14001 . DOI: 10.1209/02955075

Bretherton CS, Blossey PN, Uchida J. 2007. Cloud droplet sedimentation, entrainment efficiency, and subtropical stratocumulus albedo. Geophys. Res. Lett. 34: L03 813. DOI: 10.1029/2006GL027648.

Burnet F, Brenguier JL. 2007. Observational study of the entrainment-mixing process in warm convective clouds. J. Atmos. Sci. 64: 1995-2011. DOI: 10.1175/JAS3928.1.

Chosson F, Brenguier JL, Schueller L. 2007. Entrainment-mixing and radiative transfer simulation in boundary layer clouds. J. Atmos. Sci. 64: 2670-2682. DOI: $10.1175 /$ JAS3975.1.

De Lozar A, Mellado JP. 2013. Direct numerical simulations of a smoke cloudtop mixing layer as a model for stratocumuli. J. Atmos. Sci. 70: 2356-2375. DOI: $10.1175 / J A S-D-12-0333.1$.

Deardorff JW. 1973. The use of subgrid transport equations in a threedimensional model of atmospheric turbulence. J. Fluids Eng. 95: 429-438. DOI: $10.1115 / 1.3447047$

Deardorff JW. 1980. Cloud top entrainment instability. J. Atmos. Sci. 37: $131-147$.

Devenish BJ, Bartelo P, Brenguier JL, Collins LR, Grabowski WW, Ijzermans RHA, Reeks MW, Vassilicos JC, Wang LP, Warhaft Z. 2012. Droplet growth in warm turbulent clouds. Q. J. R. Metorol. Soc. 138: 1401-1429. DOI: 10.1002/qj.1897.

Emanuel KA. 1994. Atmospheric Convection. Oxford University Press: Oxford, UK.

Faloona I, Lenschow DH, Campos T, Stevens B, van Zaten M, Blomquist B, Thornton D, Bandy A, Gerber H. 2005. Observations of entrainment in eastern Pacific marine stratocumulus using three conserved scalars. J. Atmos. Sci. 62: 3268-3285.

Gerber H, Frick G, Malinowski SP, Brenguier JL, Burnet F. 2005. Holes and entrainment in stratocumulus. J. Atmos. Sci. 62: 443-459.

Grabowski WW, Smolarkiewicz PK. 1990. Monotone finite-difference approximations to the advection-condensation problem. Mon. Weather Rev. 118: 2082-2097. DOI: 10.1175/1520-0493(1990)118.

Grabowski WW, Wang LP. 2013. Growth of cloud droplets in a turbulent environment. Ann. Rev. Fluid Mech. 45: 293-324. DOI: 10.1146/annurevfluid-011212-140750.

Haman KE, Malinowski SP, Kurowski MJ, Gerber H, Brenguier JL. 2007. Small scale mixing processes at the top of a marine stratocumulus - a case study. Q. J. R. Meteorol. Soc. 133: 213-226. DOI: 10.1002/qj.5.

Heus T, van Heerwaarden CC, Jonker HJJ, Siebesma AP, Axelsen S, van den Dries K, Geoffroy O, Moene AF, Pino D, de Roode SR, Vila-Guerau de Arellano J. 2010. Formulation of the Dutch Atmospheric Large-Eddy Simulation (DALES) and overview of its applications. Geosci. Model Dev. 3: 415-444. DOI: 10.5194/gmd-3-415-2010.

Kumar B, Schumacher J, Shaw RA. 2012. Extreme responses of a coupled scalar-particle system during turbulent mixing. New J. Phys. 140: 115020. DOI: $10.1088 / 1367-2630$.

Kumar B, Schumacher J, Shaw RA. 2013. Cloud microphysical effects of turbulent mixing and entrainment. Theor. Comput. Fluid Dyn. 27: 361-376. DOI: 10.1007/s00162-012-0272-z.

Kurowski MJ, Malinowski SP, Grabowski WW. 2009. A numerical investigation of entrainment and transport within a stratocumulus-topped boundary layer. Q. J. R. Meteorol. Soc. 135: 77-92. DOI: 10.1002/qj.354.

Lanotte AS, Seminara A, Toschi F. 2009. Cloud droplet growth by condensation in homogeneous isotropic turbulence. J. Atmos. Sci. 66: 1685-1697. DOI: 10.1175/2008JAS2864.1

Marble FE. 1970. Dynamics of dusty gases. Ann. Rev. Fluid Mech. 2: 397-446.

Maxey MR, Riley JJ. 1983. Equation of motion for a small rigid sphere in a nonuniform flow. Phys. Fluids 26: 883. DOI: 10.1063/1.864230.
Mellado JP. 2010. The evaporatively driven cloud-top mixing layer. J. Fluid Mech. 660: 5-36. DOI: 10.1017/S0022112010002831.

Mellado JP, Stevens B, Schmidt H, Peters N. 2009. Buoyancy reversal in cloud-top mixing layers. Q. J. R. Meteorol. Soc. 135: 333-365. DOI: 10.1002/qj.417.

Mellado JP, Stevens B, Schmidt H, Peters N. 2010a. Probability density functions in the cloud-top mixing layer. New J. Phys. 12: 085010.

Mellado JP, Stevens B, Schmidt H, Peters N. 2010b. Two-fluid formulation of the cloud-top mixing layer for direct numerical simulation. Theor. Comput. Fluid Dyn. 24: 511-536. DOI: 10.1007/s00162.010.0182.x.

Moin P, Mahesh K. 1998. Direct numerical simulation: A tool in turbulence research. Ann. Rev. Fluid Mech. 30: 539-578. DOI 10.1146/annurev.fluid.30.1.539.

Pantano C, Shotorban B. 2007. Least-squares dynamic approximation method for evolution of uncertainty in initial conditions of dynamical systems. Phys. Rev. E 76: 066 705. DOI: 10.1103/PhysRevE.76.066705.

Petterssen S. 1938. On the causes and the forecasting of the California fog. Bull. Am. Meteorol. Soc. 19: 49-55.

Pruppacher HR, Klett JD. 1997. Microphysics of Clouds and Precipitation. Kluwer Academic Publishers: Dordrecht.

Randall DA. 1980. Conditional instability of the first kind upside-down. J. Atmos. Sci. 37: 125-130.

Rogers RR, Yau MK. 1989. A Short Course in Cloud Physics, third edn. Elsevier: Amsterdam.

Seifert A, Beheng KD. 2006. A two-moment cloud microphysics parameterization for mixed-phase clouds. Part 1: Model description. Meteorol. Atmos. Phys. 92: 45-66. DOI: 10.1007/s00703-005-0112-4.

Shaw RA. 2003. Particle-turbulence interactions in atmospheric clouds. Ann. Rev. Fluid Mech. 35: 183-227.

Shy SS, Breidenthal RE. 1990. Laboratory experiments on the cloudtop entrainment instability. J. Fluid Mech. 214: 1-15. DOI: $10.1017 /$ S0022112090000015.

Siems ST, Bretherton CS. 1992. A numerical investigation of cloud-top entrainment instability and related experiments. Q. J. R. Metorol. Soc. 118: $787-818$.

Smagorinsky J. 1963. General circulation experiments with the primitive equations I. The basic experiment. Mon. Weather Rev. 91: 99-164. DOI: 10.1175/1520-0493(1963)091.

Stevens B, Lenschow DH, Faloona I, Moeng C, Lilly DK, Blomquist B, Vali G, Bandy A, Campos T, Gerber H, Haimov S, Morley B, Thornton D. 2003. On entrainment rates in nocturnal marine stratocumulus. Q. J. R. Meteorol. Soc. 129: 3469-3493. DOI: 10.1256/qj.02.202.

Vaillancourt PA, Yau MK. 2000. Review of particle-turbulence interactions and consequences for cloud physics. Bull. Am. Meteorol. Soc. 81: 285-298. DOI: 10.1175/1520-0477(2000)081.

Vaillancourt PA, Yau MK, Grabowski WW. 2001. Microscopic approach to cloud droplet growth by condensation. Part I: Model description and results without turbulence. J. Atmos. Sci. 58: 1945-1964. DOI: 10.1175/15200469(2001)058.

Vaillancourt PA, Yau MK, Bartelo P, Grabowski WW. 2002. Microscopic approach to cloud droplet growth by condensation. Part II: Turbulence, clustering, and condensational growth. J. Atmos. Sci. 59: 3421-3435. DOI: 10.1175/1520-0469(2002)059.

Vincenti WG, Kruger CH. 1967. Introduction to Physical Gas Dynamics. Wiley: New York.

Williams FA. 1985. Combustion Theory, 2 edn (ch. 11). Addison-Wesley Publishing Company: Redwood City, CA.

Wood R. 2012. Stratocumulus clouds. Mon. Weather Rev. 140: 2373-2423.

Ziemer C, Wacker U. 2012. Parameterization of the sedimentation of raindrops with finite maximum diameter. Mon. Weather Rev. 140: 1589. DOI: 10.1175/MWR-D-11-00020.1 